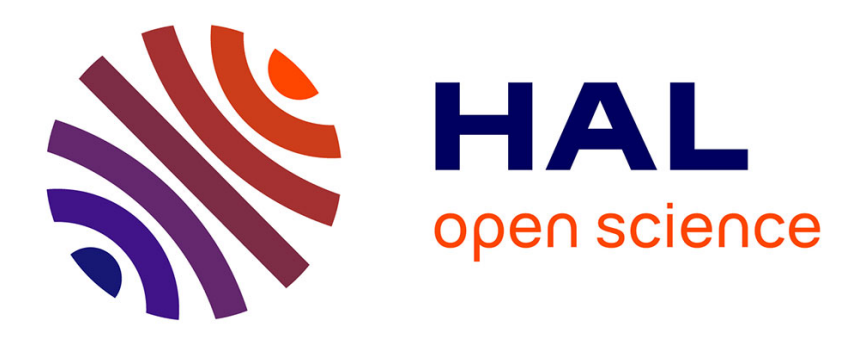

\title{
Akhmediev breather evolution in optical fiber for realistic initial conditions
}

\author{
M. Erkintalo, G. Genty, B. Wetzel, J.M. Dudley
}

\section{To cite this version:}

M. Erkintalo, G. Genty, B. Wetzel, J.M. Dudley. Akhmediev breather evolution in optical fiber for realistic initial conditions. Modern Physics Letters A, 2011, 375 (19), pp.2029-2034. 10.1016/j.physleta.2011.04.002 . hal-00605272

\section{HAL Id: hal-00605272 \\ https://hal.science/hal-00605272}

Submitted on 26 Apr 2021

HAL is a multi-disciplinary open access archive for the deposit and dissemination of scientific research documents, whether they are published or not. The documents may come from teaching and research institutions in France or abroad, or from public or private research centers.
L'archive ouverte pluridisciplinaire HAL, est destinée au dépôt et à la diffusion de documents scientifiques de niveau recherche, publiés ou non, émanant des établissements d'enseignement et de recherche français ou étrangers, des laboratoires publics ou privés. 


\title{
Akhmediev breather evolution in optical fiber for realistic initial conditions
}

\author{
M. Erkintalo ${ }^{\mathrm{a}, *}$, G. Genty ${ }^{\mathrm{a}}$, B. Wetzel $^{\mathrm{b}}$, J.M. Dudley $^{\mathrm{b}}$ \\ a Tampere University of Technology, Optics Laboratory, FI-33101 Tampere, Finland \\ b Université de Franche-Comté, Institut FEMTO-ST, UMR CNRS 6174, 25030 Besançon, France
}

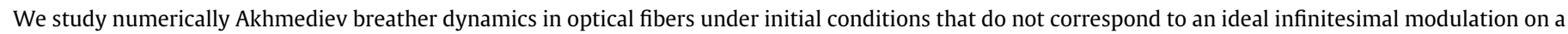

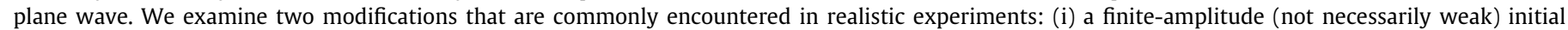

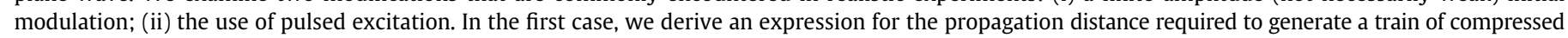

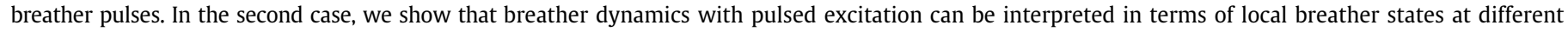
points on the pulse envelope.

\section{Introduction}

Modulation instability (MI) is a central process of nonlinear dynamics associated with the exponential amplification of a periodic modulation on a continuous wave (CW) background. Originally studied in the context of hydrodynamics [1] and plasma physics [2], it has attracted particular attention in fiber optics [3] where it has been applied to generate high contrast pulse trains [4-12]. Studies of MI have been performed using different techniques: direct numerical simulations [4]; linear stability analysis of the perturbation growth [5]; or a description of the dynamics in terms of energy exchange between a truncated number of the frequency modes generated during the amplification process [13]. However, results in the applied mathematics literature over 25 years ago have actually shown that MI can be described analytically by directly solving the integrable $(1+1) \mathrm{D}$ nonlinear Schrödinger equation (NLSE) [14-16]. This yields an exact analytic description of an ideal growth and decay cycle associated with the growth of an infinitesimal perturbation on a plane wave towards a compressed "pulse train". The evolving pulses are now generally referred to as Akhmediev breathers (ABs).

This result has been recently shown to provide an extremely powerful description of MI under a range of conditions, providing insight into the initial stage of supercontinuum (SC) generation in the anomalous dispersion regime with long pump pulses [17], and allowing the design of experiments exciting the rational Peregrine soliton solution of the NLSE $[18,19]$. These recent studies, however, have not considered in detail the wider accuracy of the AB descrip-

\footnotetext{
* Corresponding author.

E-mail address: miro.erkintalo@tut.fi (M. Erkintalo).
}

tion of MI under realistic experimental conditions, and thus the aim of this Letter is to complete this description. Specifically, we derive an analytic approximation to the evolving $A B$ field far from the maximum compression point of the breather, and this allows us to: (i) determine the experimental input conditions to excite ABs (and compressed pulse trains) whose characteristics are fixed by the initial modulated field; (ii) predict accurately the propagation distance for an input field at arbitrary modulation frequency to evolve to the compressed $\mathrm{AB}$. We also consider the accuracy of the $A B$ description using pulsed rather than $C W$ excitation and show that the evolution of a weakly-modulated pulse can be described in terms of "localized" breather states whose analytic properties are fully determined by the local power at the corresponding point on the initial pulse envelope.

\section{Excitation of breathers and distance of maximal compression}

\subsection{The Akhmediev breather formalism}

We begin by briefly reviewing the $A B$ formalism in an optical fiber context $[14,15,17]$. We consider the evolution of the field envelope $A(z, T)$ governed by the NLSE [5]:

$i \frac{\partial A}{\partial z}-\frac{\beta_{2}}{2} \frac{\partial^{2} A}{\partial T^{2}}+\gamma|A|^{2} A=0$.

Here $A(z, T)$ is normalized such that $|A(z, T)|^{2}$ represents the instantaneous power in watts. The group-velocity dispersion parameter $\beta_{2}<0$ and nonlinear coefficient $\gamma$ have dimensions of $\mathrm{ps}^{2} \mathrm{~m}^{-1}$ and $\mathrm{W}^{-1} \mathrm{~km}^{-1}$, respectively. The AB solutions to the NLSE describe a single growth-return cycle of a modulated plane wave. For a 


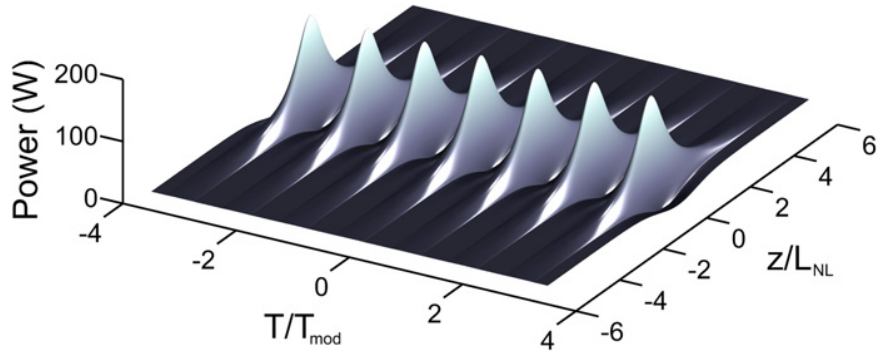

Fig. 1. Typical growth-return cycle characteristic for an Akhmediev breather. $a=$ 0.25 .

plane wave power $P_{0}$ and modulation frequency $\omega_{\text {mod }}$, the solution is:

$$
\begin{aligned}
A\left(z^{\prime}, T\right)= & \sqrt{P_{0}}\left[\frac{(1-4 a) \cosh \left(b z^{\prime}\right)+i b \sinh \left(b z^{\prime}\right)}{\sqrt{2 a} \cos \left(\omega_{\bmod } T\right)-\cosh \left(b z^{\prime}\right)}\right. \\
& \left.+\frac{\sqrt{2 a} \cos \left(\omega_{\bmod } T\right)}{\sqrt{2 a} \cos \left(\omega_{\bmod } T\right)-\cosh \left(b z^{\prime}\right)}\right] e^{i z^{\prime}},
\end{aligned}
$$

where the coefficients $a$ and $b$ are related to the modulation frequency and fiber parameters as $2 a=1-\left(\omega_{\bmod } / \omega_{c}\right)^{2}$ and $b=$ $\sqrt{8 a(1-2 a)}$ with $\omega_{c}^{2}=4 \gamma P_{0} /\left|\beta_{2}\right|$. Note that in writing Eq. (2) we have introduced a normalized distance $z^{\prime}=z / L_{N L}$, where $L_{N L}=$ $\left(\gamma P_{0}\right)^{-1}$ is the nonlinear length. Eq. (2) is valid for modulation frequencies experiencing nonzero MI gain such that $0<a<1 / 2$, i.e. $\omega_{c}>\omega_{\text {mod }}>0$. The parameter $b>0$ governs the growth rate of MI and maximum gain $b=1$ occurs for $a=1 / 4$, corresponding to $\omega_{\text {mod }}=\omega_{c} / \sqrt{2}[5]$. The AB solution exhibits a single growth-return cycle over $-\infty<z<+\infty$ as is illustrated in Fig. 1 for $a=1 / 4$ where the exponential growth and decay of a periodic train of pulses with temporal separation $T_{\bmod }=2 \pi / \omega_{\bmod }$ can readily be observed.

\subsection{Approximate input conditions and compression distance}

The amplitude and the degree of temporal localization (i.e. compression) of the $\mathrm{AB}$ peaks is maximum at $z^{\prime}=0$, and the modulated field asymptotically approaches a plane wave when $\left|z^{\prime}\right| \rightarrow \pm \infty[14,15]$. Although previous numerical studies suggested that the $A B$ evolution in Fig. 1 can be observed over a wide range of initial modulation conditions [17], we study this more quantitatively here via a straightforward Taylor series expansion of the $A B$ solution (2) for large $\left|z^{\prime}\right|$ (see Appendix A for details). In particular, retaining only the first-order terms and dropping a constant phase term that affects all terms in the expansion equally, this yields:

$A\left(z^{\prime} \rightarrow \pm \infty, T\right) \approx \sqrt{P_{0}}\left[1+2 b e^{-b\left|z^{\prime}\right|} e^{-i \psi_{ \pm}} \cos \left(\omega_{\bmod } T\right)\right.$,

where $\psi_{ \pm}= \pm \tan ^{-1}(b /(2-4 a))= \pm \tan ^{-1} \sqrt{2 a /(1-2 a)}$. It is clear that Eq. (3) has the approximate form of a weakly-modulated CW field

$A\left(z^{\prime} \rightarrow \pm \infty, T\right) \approx \sqrt{P_{0}}\left[1+a_{m o d} e^{-i \psi_{ \pm}} \cos \left(\omega_{\text {mod }} T\right)\right.$,

where we define a real modulation amplitude $a_{\text {mod }}=2 b e^{-b\left|z^{\prime}\right|}$. The phase $\psi_{ \pm}$represents the relative phase difference between the pump and modulation sidebands at $+\infty$ and $-\infty$, respectively. The relationship with the phase-matching condition for the associated four-wave mixing process is discussed in Appendix B. On one hand, the modulation amplitude $a_{\text {mod }}$ depends both on the frequency parameter $a$ and the distance $\left|z^{\prime}\right|$ from the expected point of maximum temporal compression. On the other hand, the phasemismatch $\psi_{ \pm}$does not depend on distance as long as the pump remains undepleted, which reflects the fact that the phase difference between the pump and the sidebands remains constant with propagation.

It is straightforward to use numerical simulations of the NLSE to determine under what conditions the approximate form of the initial condition described by Eq. (4) leads to the ideal AB growthreturn evolution as seen in Fig. 1. This is of course an important study to carry out as the synthesis of an input field having the form of Eq. (4) is experimentally straightforward. The results of extensive simulations over the full range of modulation frequencies $0<a<0.5$ indicate that provided the effective modulation amplitude satisfies $a_{\text {mod }}<0.2$, the approximate form of the initial condition yields the expected $\mathrm{AB}$ evolution dynamics. In practical terms, what this means is that injecting a field with the form of Eq. (4) with $a_{\text {mod }}<0.2$ into an optical fiber results in an evolution trajectory similar to that of an ideal $\mathrm{AB}$ over the first growth-return cycle. We emphasize of course that such an initial condition remains an approximation of the exact analytical $A B$ which will lead to deviation from the perfect decay of the $A B$ solution and recurrence. However, this is generally not important for applications aiming to use MI for pulse train generation where it is the pulse characteristics at the first point of compression in the fiber that are of interest. Although such a periodic evolution may be of fundamental interest due to its links with the Fermi-Pasta-Ulam recurrence phenomenon [20,21], it is beyond the scope of this Letter and here we have restricted our discussion to the first growth-return cycle.

Simulations using the approximate initial condition are shown in Fig. 2(a) for $a=0.25$ and $a_{\text {mod }}=0.02$. The false color plot on the top shows the growth-return cycle evolution of the field intensity and the bottom plot shows a comparison between the simulated intensity profiles at the distance of maximal compression with that of the exact analytical $A B$. We can see how the two intensity profiles are in excellent agreement at the distance of maximum compression. In fact, we have checked that we observe similar agreement at any distance $z^{\prime}$ and across the full MI gain curve for any value of the normalized frequency parameter $0<a<1 / 2$.

The distance scale used in this figure is such that the position $z^{\prime}=0$ corresponds to the input point in the fiber and not the point of maximal compression as for the ideal $\mathrm{AB}$ solution. But in fact, this illustrates clearly how we can use the relation $a_{\text {mod }}=2 b e^{-b\left|z^{\prime}\right|}$ to determine the distance from an input field to the point of compression for any initial modulation amplitude through the remarkably simple result:

$z_{0}=-\frac{L_{N L}}{b} \ln \left(\frac{a_{m o d}}{2 b}\right)$.

In fact, a more approximate form for this characteristic compression distance has been obtained by other workers [22,23] assuming purely exponential instability growth, but the result here is significantly more accurate, differing by a factor of $2 b$ in the argument of the log-function. For $a_{\text {mod }}=0.02$, we compare in Fig. 3 predictions of the compression distance using Eq. (5) (black line) with results from NLSE simulations (red circles) over the full range of modulation frequency parameter $a$ and we see that there is excellent agreement. We note that similar results to those shown in the figure are obtained for larger modulation amplitudes up to $a_{\text {mod }}<0.2$. For completeness we also plot in Fig. 3 the optimal distance of pulse train generation from seeded-MI dynamics derived in $[22,23]$. It is clear that the distance of maximum compression as derived from the $A B$ formalism provides a significantly improved estimate. This can be understood from the fact that the $A B$ formalism accounts for both the pump depletion and phase-mismatch between the pump and sidebands unlike in the exponential amplification model. 


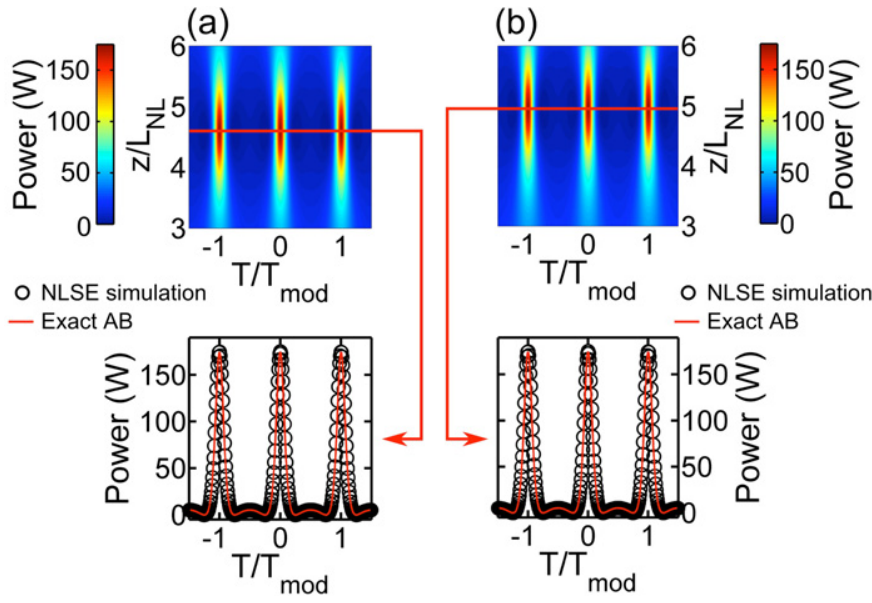

Fig. 2. (a) Top: Simulated temporal evolution of an input field corresponding to Eq. (4) for $a=0.25$ and $a_{\text {mod }}=0.02$. Bottom: Comparison of the simulated intensity profile at the distance of maximum compression with that of the corresponding exact AB. (b) Same as (a) but for an input field with $\psi=0$. The numerical simulations use standard single-mode fiber parameters at $1550 \mathrm{~nm}$, with $\beta_{2}=-20 \mathrm{ps}^{2} \mathrm{~km}^{-1}$, $\gamma=1.1 \mathrm{~W}^{-1} \mathrm{~km}^{-1}$, and we assume $P_{0}=30 \mathrm{~W}$. The nonlinear length for the chosen parameters corresponds to $L_{N L}=30.3 \mathrm{~m}$. The time scale is plotted in terms of the oscillation period $T_{\text {mod }}=2 \pi / \omega_{\text {mod }}$.

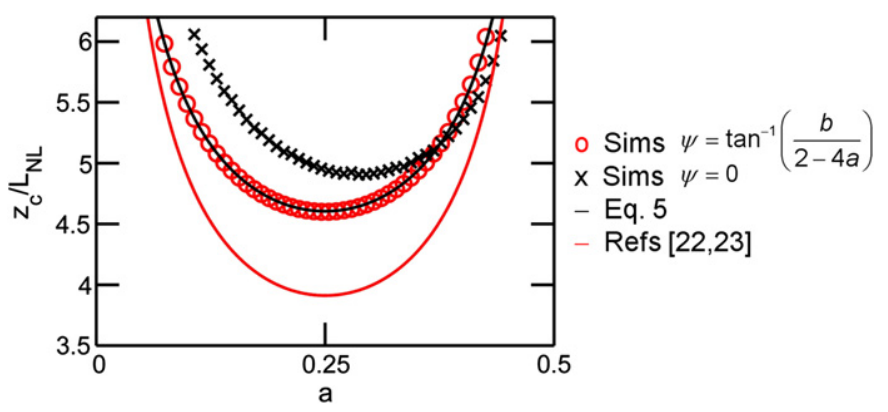

Fig. 3. Maximum compression distance vs. modulation frequency parameter $a$ as obtained from Eq. (5) (black solid line). Distance obtained from numerical NLSE simulations for a weakly-modulated input field with initial phase $\psi=\tan ^{-1}(b /(2-$ $4 a)$ ) (red circles) and initial phase $\psi=0$ (black crosses) are also plotted. The red solid line indicates the distance derived in Refs. $[22,23]$. In all cases $a_{\bmod }=0.02$ and other parameters as those used for Fig. 2. (For interpretation of the references to color in this figure legend, the reader is referred to the web version of this Letter.)

An important factor in the solution given by Eq. (4) is the presence of the relative pump-sideband phase difference $\psi$. Although in some experiments, it may be possible to control this accurately to obtain precise correspondence with the theoretical $A B$ result, it is interesting to examine how the dynamics change if we use a purely real input field of the form $\sqrt{P_{0}}\left[1+a_{\text {mod }} \cos \left(\omega_{\bmod } T\right)\right]$ (i.e. with $\psi=0$ ). For this purpose the upper subplot in Fig. 2(b) shows the intensity evolution as in the ideal case in Fig. 2(a) but with $\psi=0$. We see similar temporal evolution to the ideal case and as shown in the lower subplot, the intensity profile at the distance of maximum compression coincides with the "maximallycompressed" AB. The exact distance at which the compression occurs, however, is different from the case in Fig. 2(a) with initial pump-sideband phase control. Physically, this difference in the compression distances is a manifestation of the fact that the growth rate of the modulation is sensitive to the relative pumpsideband phase difference [5] (see also Appendix B). Yet, simulation results as shown in Fig. 3 carried out over the full range of $a$ shows that this compression distance offset is $\leqslant 15 \%$. This suggests that Eq. (5) provides a very useful result allowing compression distance to be estimated even when $\psi=0$.

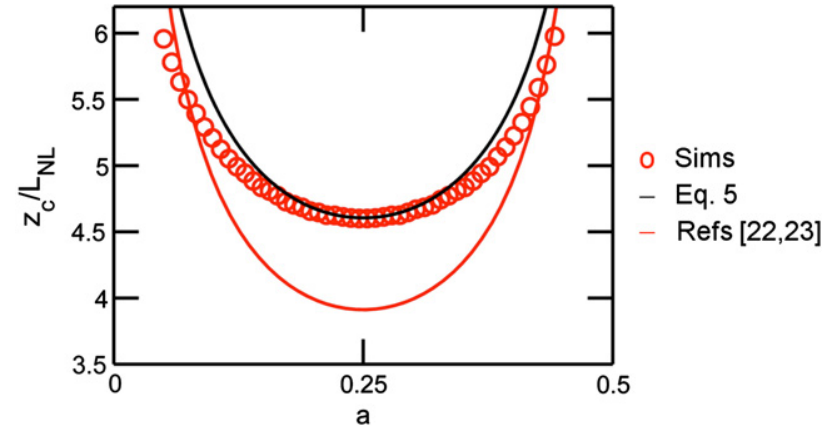

Fig. 4. Maximum compression distance vs. modulation frequency parameter $a$ as obtained from Eq. (5) (black solid line). Distance obtained from numerical NLSE simulations for a weakly-modulated input field with a single sideband (see Eq. (6)) (red circles). The red solid line indicates the distance derived in Refs. [22,23]. In all cases $a_{m o d}=0.02$ and other parameters as those used for Fig. 2. (For interpretation of the references to color in this figure legend, the reader is referred to the web version of this Letter.)

\subsection{Dynamics with only single sideband excitation}

Synthesizing an input field as given by Eq. (4) would require an appropriate amplitude modulation technique or the use of a pump and two distinct sideband seeds with phase-control. In some experiments, an alternative approach could use an input with a single sideband that can be arbitrarily tuned with respect to the pump. This would correspond to an experimental arrangement that employs e.g. two CW lasers as typically used in seeded-MI experiments for generating ultra-fast pulse trains.

This particular case has an input field given by:

$A(0, T)=\sqrt{P_{0}}\left[1+a_{\text {mod }} e^{i\left(\omega_{\text {mod }} T+\psi\right)}\right.$.

Even for this single-sideband input, apart from a trivial temporal translation the field evolution closely resembles that of the ideal $\mathrm{AB}$ determined by the corresponding modulation frequency parameter as fixed by the initial pump-sideband frequency detuning. Indeed, in this case the symmetrical sideband with respect to the pump is generated at a very early propagation stage due to parametric four-wave mixing and the phase of the sideband is automatically set such that the initial pump-sideband phase mismatch (as defined in Appendix B) $\theta=-\pi / 2$ [5]. As a consequence, in contrast with a dual-sideband input the evolution is independent of the input pump-sideband phase-mismatch $\psi$ which, from an experimental view point means, that an active stabilization of the phase difference is not required to excite an $A B$ with this technique. It is worth noting that although the pump-sideband phase mismatch $\theta=-\pi / 2$ is valid only for an $A B$ whose modulation parameter $a=0.25$ (see Appendix B), the distance of maximum compression for a single sideband input is well approximated by Eq. (5) for arbitrary $a$ as illustrated in Fig. 4. Therefore Eqs. (2), (5), and (6) provide a simple general recipe to generate ultra-fast pulse trains with desired characteristics. Finally, we point out that, although we have restricted ourselves to the pure NLSE, we have carefully checked that the inclusion of higher-order effects such as stimulated Raman scattering, higher-order dispersion and selfsteepening do not modify significantly the dynamics during the first growth cycle and, therefore, the results are also applicable in the presence of higher-order perturbations.

\section{The use of a pulsed input - non-CW initial conditions}

In many experiments studying MI, the power levels needed to seed the instability are such that it is necessary to use high peak power narrowband pulses from modelocked lasers rather than an ideal CW input field [24-27]. It is clear that the dynamics here will 
(a)

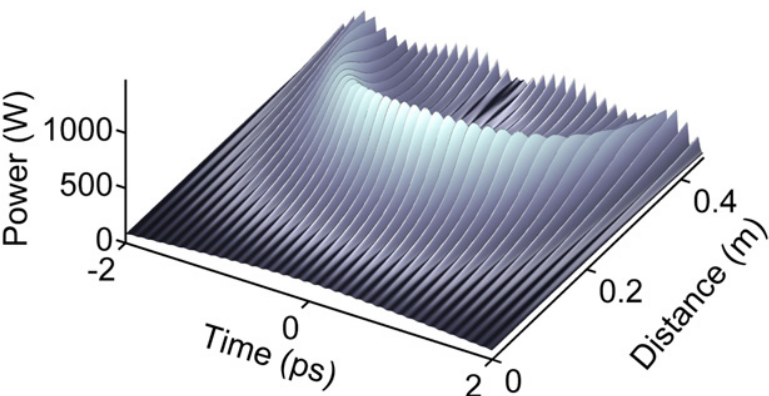

(b)

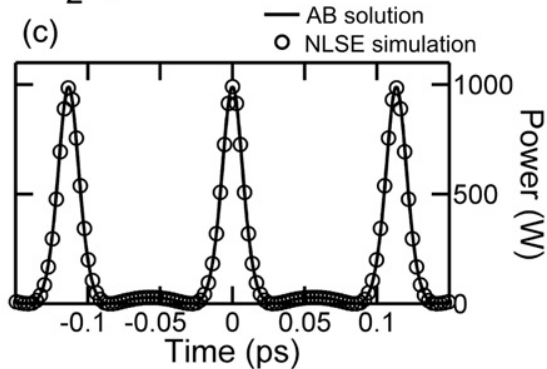

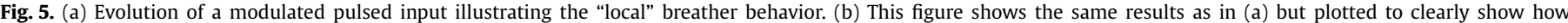

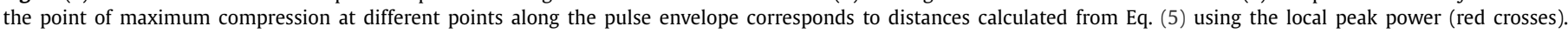

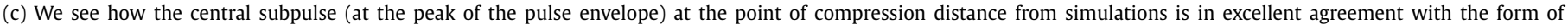
the compressed pulse profile from $\mathrm{AB}$ theory. (For interpretation of the references to color in this figure legend, the reader is referred to the web version of this Letter.)

not be exactly the same as expected from the $A B$ theory, because there will be power variation across the pulse envelope. In this section we consider how the analytic $A B$ theory above applies to this particular case of non-ideal (yet realistic) initial conditions.

In order to examine how the $\mathrm{AB}$ formalism can be applied to a pulsed input we numerically study the evolution of a weakly modulated pulsed field of the form

$A(0, T)=\sqrt{P_{0}} \operatorname{sech}\left(T / T_{0}\right)\left[1+a_{\text {mod }} e^{i \psi} \cos \left(\omega_{\bmod } T\right)\right.$,

where $P_{0}$ and $T_{0}$ represent the peak power and duration of the unmodulated pulse envelope. From an experimental point of view this initial condition corresponds to the simultaneous injection of a strong pump pulse and frequency-shifted seed replicas by an amount $\omega_{\bmod }$ with a $\psi$ phase difference between the pump and the seed pulses. The full-width at half-maximum of the pump and seed pulses is related to the parameter $T_{0}$ through $\tau=1.763 T_{0}$. It is clear that unambiguous MI dynamics in the pulsed regime will be seen only if the pulse duration significantly exceeds the modulation period $\tau \gg T_{\text {mod }}$.

Numerical results studying the evolution of a 4 ps (FWHM), $170 \mathrm{~W}$ peak power weakly modulated pulse $\left(a_{\text {mod }}=0.02\right)$ are shown in Fig. 5. Here we use realistic parameters of a commercially available photonic crystal fiber (Crystal Fiber NL-PM-750) at $815 \mathrm{~nm}: \beta_{2}=-13.7 \mathrm{ps}^{2} \mathrm{~km}^{-1}$ and $\gamma=0.12 \mathrm{~W}^{-1} \mathrm{~m}^{-1}$. The initial phase $\psi$ is taken from the exact $A B$ solution as calculated from the corresponding $a$ parameter. The modulation frequency $\omega_{\text {mod }}=55 \mathrm{THz}$ corresponds to the maximum of the MI gain curve for the power level at the peak of the pulse. With this choice of parameters $T_{\text {mod }}=114 \mathrm{fs}$, satisfying the requirement where we would expect MI dynamics even with a pulsed input, as discussed above.

With a pulsed input, comparing Fig. 1 with Fig. 5(a) clearly shows that the growth of the modulation does not occur uniformly across the pulse profile. Certainly we see that propagation yields increased modulation contrast and sub-pulse formation across the pulse envelope, but the point of maximum amplitude and temporal compression occurs at different propagation distances for different temporal points across the envelope. This behaviour, however, can be understood very simply in terms of "localized" breather states, where the local peak power at any point on the pulse envelope determines the breather properties at this point. Specifically, a modulation cycle at a given temporal position $T_{n} \approx \pm n T_{\text {mod }}$, $n=0,1,2, \ldots$, along the pulse envelope will evolve with $A B$ characteristics of a CW wave of power $P_{n}=P_{0} \operatorname{sech}^{2}\left(T_{n} / T_{0}\right)$. These "local" $A B$ dynamics are highlighted in Fig. 5(b) where the maximally compressed distances of separate sub-peaks are shown to closely follow those analytically predicted using Eq. (5) when accounting for the power variation along the pulse envelope. To further illustrate the correspondence with ideal $A B$ dynamics we show in Fig. 5(c) the temporal profile of the central sub-peak at its maximally-compressed distance which is exactly fitted by the ideal $A B$ sub-peak corresponding to the peak power of the unmodulated pulse. Of course, we have carefully checked that the sub-pulses at the wings of the pulse envelope are equally well fitted with an isolated sub-pulse of the $A B$ whose power is determined by the corresponding point along the input pulse envelope.

As with the CW case, the pulsed dynamics with a single initial sideband seed are similar to that observed with dual sideband input and the local compression distances are well predicted by Eq. (5). However, it should be noted that for a fixed modulation frequency $\omega_{\bmod }$ the modulation parameter $a$ decreases as we move from the peak of the pulse envelope to the wings, which correspondingly leads to discrepancies when using Eq. (5) to predict the distance of maximal compression for the wings of the pulse in the case of a single sideband seed (see Fig. 4).

\section{Conclusions}

There are several important conclusions to be drawn from this work. We have used the Akhmediev breather formalism to derive an improved analytical prediction for the distance of maximal compression of a weakly modulated CW field and shown that the theory can be extended to the pulsed regime. Specifically, we have found that the evolution of a weakly modulated pulse can be depicted in terms of "local" breather states, whose analytic properties are determined by the local power at the corresponding point on the initial pulse envelope. We expect our results to be of use in the design and realization of ultrahigh-repetition-rate sources as 
well as to provide significant insight into the initial stages of noisedriven SC generation.

\section{Appendix A}

A first-order approximation of the $A B$ solution (see Eq. (2)) at large distances from the maximum compression point can be obtained by keeping only the dominant term in the hyperbolic trigonometric functions

$\cosh \left(b z^{\prime}\right) \approx \frac{e^{b\left|z^{\prime}\right|}}{2}$

and

$\sinh \left(b z^{\prime}\right) \approx \pm \frac{e^{b\left|z^{\prime}\right|}}{2}$

where the ' + ' and ' - ' sign corresponds to $z^{\prime}=\infty$ and $z^{\prime}=-\infty$, respectively. Factorizing out $e^{b\left|z^{\prime}\right|}$ from the numerator and denominator the $\mathrm{AB}$ solution can be written as

$$
\begin{aligned}
& A B\left(z^{\prime} \rightarrow \pm \infty, T\right) \\
& \quad \approx \sqrt{P_{0}}\left[\frac{(1-4 a) \mp i b-2 \sqrt{2 a} e^{b\left|z^{\prime}\right|} \cos \left(\omega_{\text {mod }} T\right)}{1-2 \sqrt{2 a} e^{b\left|z^{\prime}\right|} \cos \left(\omega_{\bmod } T\right)}\right] e^{i z^{\prime}} .
\end{aligned}
$$

Expanding the denominator in Taylor-series and retaining only the first term, we obtain

$$
\begin{aligned}
& A B\left(z^{\prime} \rightarrow \pm \infty, T\right) \\
& \approx \sqrt{P_{0}}[(4 a-1) \mp i b \\
& \quad+(4 a-2 \mp i b) 2 \sqrt{2 a} e^{-b\left|z^{\prime}\right|} \cos \left(\omega_{\bmod } T\right) e^{i z^{\prime}} .
\end{aligned}
$$

After some straightforward algebra the $A B$ solution is given by

$$
\begin{aligned}
& A B\left(z^{\prime} \rightarrow \pm \infty, T\right) \\
& \quad \approx \sqrt{P_{0}}\left[1+2 b e^{-b\left|z^{\prime}\right|} e^{\mp i \tan ^{-1}\left(\frac{b}{2-4 a}\right)} \cos \left(\omega_{\bmod } T\right) e^{\mp i \phi} e^{i z^{\prime}},\right.
\end{aligned}
$$

where $\phi=\tan ^{-1}\left(\frac{b}{4 a-1}\right)+\pi$ for $a \leqslant 0.25$ and $\phi=\tan ^{-1}\left(\frac{b}{4 a-1}\right)$ otherwise. After dropping the phase term that affects all the terms in the brackets equally (and does not affect the evolution of the field intensity), the solution can be conveniently written as

$A B\left(z^{\prime} \rightarrow \pm \infty, T\right) \approx \sqrt{P_{0}}\left[1+2 b e^{-b\left|z^{\prime}\right|} e^{-i \psi_{ \pm}} \cos \left(\omega_{\bmod } T\right)\right.$

where $\psi_{ \pm}= \pm \tan ^{-1}(b /(2-4 a))$.

\section{Appendix B}

Connection between $\mathrm{ABs}$ and degenerate four-wave mixing (FWM) in the undepleted pump approximation can be derived by considering degenerate FWM processes between the pump and sidebands of the initial modulated signal. The dynamical evolution of the system can be solved by injecting in the NLSE a 3-frequency field of the form

$$
A(z, T)=a_{0}(z)+a_{1}(z) e^{-i \omega_{\text {mod }} T}+a_{2}(z) e^{+i \omega_{\bmod } T},
$$

where $\omega_{\text {mod }}$ represent the applied modulation frequency and $a_{0}$, $a_{1}$, and $a_{2}$ represent the complex amplitude of the pump, Stokes, and anti-Stokes frequency components, respectively. Separating the terms oscillating at different frequencies yields three coupled differential equations which, in the undepleted pump approximation (i.e. assuming $\left|a_{1}\right|^{2},\left|a_{2}\right|^{2} \ll\left|a_{0}\right|^{2}$ ), can be written as $i \frac{d a_{0}}{d z}+\gamma\left|a_{0}\right|^{2} a_{0}=0$

$i \frac{d a_{1}}{d z}+\frac{\beta_{2}}{2} \omega_{\text {mod }}^{2} a_{1}+2 \gamma\left|a_{0}\right|^{2} a_{1}+\gamma a_{0}^{2} a_{2}^{*}=0$,

$i \frac{d a_{2}}{d z}+\frac{\beta_{2}}{2} \omega_{\text {mod }}^{2} a_{2}+2 \gamma\left|a_{0}\right|^{2} a_{2}+\gamma a_{0}^{2} a_{1}^{*}=0$.

Writing the complex amplitudes $a_{k}(z)=b_{k}(z) e^{i \phi_{k}(z)}$ with $b_{k}$ and $\phi_{k}$ real amplitude and phase, respectively, we find that the evolution of the Stokes complex amplitude is governed by

$$
\begin{aligned}
& \frac{d b_{1}}{d t}+i \frac{d \phi_{1}}{d z} b_{1} \\
& \quad=i\left(\frac{\beta_{2}}{2} \omega_{\text {mod }}^{2}+2 \gamma\left|a_{0}\right|^{2}\right) b_{1}+i \gamma b_{0}^{2} b_{2} e^{i\left(2 \phi_{0}-\phi_{2}-\phi_{1}\right)} .
\end{aligned}
$$

Introducing the pump-sidebands linear phase-mismatch $\theta=2 \phi_{0}-$ $\phi_{2}-\phi_{1}$ and separating the real and imaginary parts we subsequently obtain for the amplitude and phase-mismatch evolution

$\frac{d b_{1}}{d z}=-\gamma b_{0}^{2} b_{2} \sin (\theta)$

$\frac{d \phi_{1}}{d z}=\kappa+\gamma \frac{b_{0}^{2} b_{2}}{b_{1}} \cos (\theta)$

where $\kappa=\frac{\beta_{2}}{2} \Omega^{2}+2 \gamma\left|a_{0}\right|^{2}$. Similar equations for the evolution of the anti-Stokes amplitude and phase can be written by interchanging indices $1 \leftrightarrow 2$. Accounting for the phase variation of the pump due to self-phase modulation as

$\frac{d \phi_{0}}{d z}=\gamma b_{0}^{2}$

and replacing $b_{k}=\sqrt{P_{k}}$, the evolution of the power and phasemismatch of the Stokes and anti-Stokes modes are described by

$\frac{d P_{1}}{d z}=\frac{d P_{2}}{d z}=-2 \gamma P_{0} \sqrt{P_{1} P_{2}} \sin (\theta)$,

$\frac{d \theta}{d z}=\left|\beta_{2}\right| \omega_{\text {mod }}^{2}-2 \gamma P_{0}-\gamma \frac{P_{0}}{\sqrt{P_{1} P_{2}}}\left(P_{1}+P_{2}\right) \cos (\theta)$

where we have assumed anomalous dispersion, i.e. $\operatorname{sgn}\left(\beta_{2}\right)=-1$. If we assume the FWM process to be seeded from an initial cosine modulation the power of the Stokes and anti-Stokes sidebands is equal so that $P_{1}=P_{2}$ and Eqs. (B.9) and (B.10) reduce to

$\frac{d P_{1}}{d z}=\frac{d P_{2}}{d z}=-2 \gamma P_{0} P_{1} \sin (\theta)$,

$\frac{d \theta}{d z}=\left|\beta_{2}\right| \omega_{\text {mod }}^{2}-2 \gamma P_{0}-2 \gamma P_{0} \cos (\theta)$.

From Eq. (B.12) it can be seen that if the initial phase-mismatch between the pump and sidebands $\theta$ is such that

$\theta= \pm \cos ^{-1}\left(\frac{\left|\beta_{2}\right| \omega_{\text {mod }}^{2}-2 \gamma P_{0}}{2 \gamma P_{0}}\right)$,

it remains clamped to this value with propagation as long as the undepleted pump approximation holds. Replacing $\omega_{\text {mod }}$ in terms of the $a$-parameter as $\omega_{\text {mod }}^{2}=4 \gamma P_{0}(1-2 a) /\left|\beta_{2}\right|$ we can rewrite the phase-mismatch condition for constant phase-mismatch evolution as

$\theta_{ \pm}= \pm \cos ^{-1}(1-4 a)$.

This condition on the phase-mismatch value as given by Eq. (B.14) is precisely equal to the phase difference between the pump and first Fourier modes as obtained from the Taylor series expansion of ABs at $\pm \infty: A\left(z^{\prime} \rightarrow \pm \infty, T\right)=\sqrt{P_{0}}\left[1+2 b e^{-j \psi_{ \pm}} e^{-b\left|z^{\prime}\right|} \cos \left(\omega_{\bmod } T\right)\right]$ with 
$\psi_{ \pm}= \pm \tan ^{-1}\left(\frac{b}{2-4 a}\right)= \pm \frac{1}{2} \cos ^{-1}(1-4 a)=\frac{1}{2} \theta_{ \pm}$,

where the ' + ' and ' - ' signs correspond to the analytical $A B$ at $+\infty$ and $-\infty$, respectively. Therefore, at large distance from the point of maximum compression the evolution of ABs can be interpreted as degenerate FWM with constant phase-mismatch.

When a single sideband is injected together with the pump, the phase of the symmetrical sideband resulting from the parametric process is automatically set such that $\theta=-\pi / 2$ which corresponds to the phase-mismatch of an $A B$ at $-\infty$ whose modulation parameter $a=0.25$.

\section{References}

[1] T.B. Benjamin, J.E. Feir, J. Fluid Mech. 27 (03) (1967) 417.

[2] T. Taniuti, H. Washimi, Phys. Rev. Lett. 21 (1968) 209.

[3] A. Hasegawa, F.W. Brinkman, IEEE J. Quantum Electron. 16 (1980) 694.

[4] A. Hasegawa, Opt. Lett. 9 (1984) 288.

[5] G.P. Agrawal, Nonlinear Fiber Optics, 4th ed., Academic Press, 2007.

[6] K. Tai, A. Tomita, J.L. Jewell, A. Hasegawa, Appl. Phys. Lett. 49 (1986) 236.

[7] D. Mestdagh, Opt. Lett. 13 (1988) 829.

[8] E.M. Dianov, P.V. Mamyshev, A.M. Prokhorov, S.V. Chernikov, Opt. Lett. 14 (1989) 1008.

[9] P.V. Mamyshev, S.V. Chernikov, E.M. Dianov, A.M. Prokhorov, Opt. Lett. 15 (1990) 1365.
[10] S.V. Chernikov, E.M. Dianov, D.J. Richardson, R.I. Laming, D.N. Payne, Appl. Phys. Lett. 63 (1993) 293.

[11] J.M. Dudley, F. Gutty, S. Pitois, G. Millot, IEEE J. Quantum Electron. 37 (4) (2001) 587.

[12] J. Fatome, S. Pitois, G. Millot, IEEE J. Quantum Electron. 42 (2006) 1038.

[13] G. Cappellini, S. Trillo, J. Opt. Soc. Am. B 8 (1991) 824.

[14] N. Akhmediev, V.I. Korneev, Theor. Math. Phys. 69 (2) (1986) 1089.

[15] N. Akhmediev, V.M. Eleonskii, N.E. Kulagn, Theor. Math. Phys. (USSR) 72 (1987) 809.

[16] N. Akhmediev, A. Ankiewicz, Solitons, Nonlinear Pulses and Beams, Chapman \& Hall, 1997.

[17] J.M. Dudley, G. Genty, F. Dias, B. Kibler, N. Akhmediev, Opt. Express 17 (2009) 21497.

[18] B. Kibler, J. Fatome, C. Finot, G. Millot, F. Dias, G. Genty, N. Akhmediev, J.M Dudley, Nature Physics 6 (2010) 790.

[19] D.H. Peregrine, J. Aust. Math. Soc. Ser. B 25 (1983) 16.

[20] E. Fermi, J. Pasta, S. Ulam, in: E. Segrè (Ed.), Collected Papers of Enrico Fermi, vol. 2, The University of Chicago, Chicago, 1965, pp. 977-988.

[21] G. Van Simaeys, Ph. Emplit, M. Haelterman, Phys. Rev. Lett. 87 (2001) 033902.

[22] S.M. Kobtsev, S.V. Smirnov, Opt. Express 16 (2008) 7428.

[23] E.R. Tracy, H.H. Chen, Phys. Rev. A 37 (1988) 815.

[24] T. Schreiber, J. Limpert, H. Zellmer, A. Tünnermann, K.P. Hansen, Opt. Commun. 228 (2003) 71.

[25] A. Demircan, U. Bandelow, Opt. Commun. 244 (2005) 181.

[26] J.M. Dudley, G. Genty, S. Coen, Rev. Mod. Phys. 78 (2006) 1135.

[27] A. Mussot, A. Kudlinski, E. Louvergneaux, M. Kolobov, M. Taki, Opt. Lett. 35 (2010) 1194. 